\title{
L'ouverture du marché du riz japonais. Comment faire accepter l'abandon de la souveraineté ?
}

Liberalization of Japanese rice market: How to make loss of sovereignty accepted?

La apertura del mercado del arroz japonés. ¿ Cómo hacer aceptar el abandono de la soberanía?

\section{Thierry Guthmann}

\section{(2) OpenEdition \\ Journals}

Édition électronique

URL : https://journals.openedition.org/mots/8293

DOI : $10.4000 /$ mots.8293

ISSN : 1960-6001

Éditeur

ENS Éditions

\section{Édition imprimée}

Date de publication : 1 mars 2003

Pagination : 103-118

ISBN : 2-84788-027-5

ISSN : 0243-6450

\section{Référence électronique}

Thierry Guthmann, «L'ouverture du marché du riz japonais. Comment faire accepter l'abandon de la souveraineté ? », Mots. Les langages du politique [En ligne], 71 | 2003, mis en ligne le 05 mai 2008, consulté le 23 avril 2022. URL : http://journals.openedition.org/mots/8293 ; DOI : https://doi.org/ $10.4000 /$ mots.8293 


\section{L'ouverture du marché du riz japonais Comment faire accepter l'abandon de la souveraineté?}

À la fin de l'année 1993, la question de l'ouverture du marché du riz japonais à la concurrence internationale était au centre des préoccupations politiques et médiatiques. Cinq années auparavant, en 1988, des négociations commerciales similaires, relatives à cette époque au marché des oranges et de la viande de bœuf, n'avaient pas suscité un intérêt aussi vif. L'analyse comparative du contenu des manchettes des principaux quotidiens nationaux d'information pendant les deux dernières semaines précédant la fin des négociations révèle un taux de traitement beaucoup plus élevé dans le cas de la question de l'ouverture du marché du riz (négociations multilatérales dites du GATT, Uruguay Round). Ceci s'explique par le fait que, à la différence des négociations sur les oranges et la viande de bœuf (négociations bilatérales États-Unis, Japon), la crise du riz de 1993 constituait plus qu'un simple enjeu commercial ou économique.

Le problème en effet était lié à la sécurité alimentaire du Japon, dont la denrée alimentaire de base, malgré la forte occidentalisation des modes alimentaires, demeurait le riz. Une politique d'autosuffisance complète était pratiquée depuis la période de la Seconde guerre mondiale. Cependant, à partir de la fin des années 1980, les pays producteurs de riz, et en particulier les États-Unis d'Amérique, commencèrent à plaider avec véhémence pour la fin de l'exception commerciale, et donc pour une ouverture du marché du riz japonais à leurs exportations. Les couts de production et à la vente du riz japonais étant beaucoup plus élevés que ceux de ses concurrents étrangers, une libéralisation du marché devait logiquement entrainer la faillite d'une bonne partie des agriculteurs de l'archipel, et par voie de conséquence, une forte augmentation du taux de dépendance alimentaire du pays. Le rôle essentiel joué par les rizières dans la régulation de l'écosystème et dans la prévention des inondations justifiait les fortes

\footnotetext{
- Université nationale, préfecture de Mie, Japon.
} 
inquiétudes nourries par la menace de leur disparition. Par ailleurs, l'attachement sentimental à cette céréale, omniprésente sous différents aspects dans la culture traditionnelle japonaise, achevait de créer un réel climat d'angoisse au sein de la population.

En effet, le riz ne peut pas être considéré comme un simple aliment. La graine présente des origines mythico-religieuses, fait partie intégrante de bon nombre de cérémonies religieuses, occupe une place centrale dans les rites de succession impériaux, rime traditionnellement avec prospérité, et a pu, sous sa forme d'aliment cuit, être considéré comme une métaphore de l'unité familiale ${ }^{1}$. Comme le pain pour la France et les Français, le riz constitue un élément majeur de la culture traditionnelle japonaise. De manière plus ou moins consciente, les menaces qui pesaient alors sur le riz furent fréquemment ressenties comme des menaces pesant sur un élément constitutif essentiel de l'identité collective.

Dans cet article, il s'agira d'examiner la manière dont toute cette émotion, suscitée par la crise du riz, fut gérée sur le plan politique et plus précisément sur le plan du discours politique. Les textes présentés ci-après sont représentatifs des quatre principaux types de discours politiques entendus à l'occasion de la crise du riz de 1993.

Le type le plus fréquent est le discours d'inspiration technocratique, mâtiné de quelques passages faisant référence à l'héritage mythico-symbolique lié au riz. Le pathos y est présent mais souvent concentré sur une portion réduite du texte. Le second type correspond à celui où le politicien se refuse à l'utilisation du pathos et ne s'appuie que sur la raison. Le troisième type est caractérisé par des développements qui se veulent objectifs mais dont le ton laisse apparaitre une émotion difficilement contenue. Le pathos y est présent, mais il est en quelque sorte involontaire. Enfin le dernier type de discours, qui fut également le moins fréquent, se présente sous la forme d'un appel équilibré à la raison et à l'émotion de l'auditoire.

Tout en décrivant et analysant ces quatre types de discours, notre objectif sera d'expliquer en quoi le quatrième type semble le plus adapté à une situation de crise, et ceci tout particulièrement lorsqu'une identité collective nationale se trouve être menacée par les avancées de la mondialisation libérale des échanges.

1. Sur tous ces points, voir l'ouvrage de E. Ohnuki-Tierney, 1993, Rice as Self (Japanese Identities through Time), Princeton, Princeton University Press; ainsi que la première partie de ma thèse de doctorat, Pour un regard anthropologique sur la part d'émotion dans les crises politiques modernes (une étude de cas: l'ouverture du marché du riz japonais), septembre 2000, Université d'Aix-Marseille 3, Institut d'Études Politiques. 
Dans les discours, le pathos se révéla très souvent sous la forme de «symboles de condensation». Murray Edelman, qui reprend le concept à Edward Sapir en donne la définition suivante:

Les symboles de condensation évoquent l'émotion associée avec la situation. Ils condensent dans un seul évènement symbolique, signe ou acte patriotique, fierté, angoisses, souvenir de gloires ou d'humiliations passées, promesses de grandeur future: l'un de ces éléments ou leur totalité ${ }^{2}$.

Cette notion de «symbole de condensation» nous aidera à localiser dans les discours les expressions à forte potentialité émotionnelle, c'est-àdire en termes de rhétorique, le pathos.

\section{Un usage limité du pathos}

Le discours qui sera analysé en premier lieu est caractérisé par un usage limité de l'émotion. Les arguments rationnels et l'énumération des faits restent prépondérants. Ainsi, dans la question au gouvernement du député conservateur Yoshirô Mori, les passages faisant appel au pathos sont comme neutralisés par la forte technicité du propos ${ }^{3}$.

Après avoir vanté la politique de son parti, le Parti libéral démocrate (PLD), alors principal parti de l'opposition, Y. Mori attaque durement le chef du Gouvernement, l'accusant de malhonnêteté vis-à-vis des citoyens, exprimant à la suite la honte qu'il éprouve à entendre des accusations de «double langage (nimai jita)» proférées à l'encontre du Premier ministre de son pays ${ }^{4}$. Y. Mori dénonce ensuite la désinvolture du chef du Gouvernement qui, selon lui, aurait confié les négociations agricoles du GATT à des fonctionnaires, ne jugeant pas utile de dépêcher le ministre des Affaires étrangères sur place, à Genève. Devant cette conduite très peu responsable du Premier ministre, alors qu'il s'agit d'un problème qui touche «l'avenir» et «l'existence» des citoyens, Y. Mori explique qu'il

2. M. Edelman, 1964, The symbolical uses of politics, Urbana, University of Illinois Press, p. 6.

3. 13 décembre 1993, Kampô (Journal officiel), gôgai, dai 128 kai kokkai, shûgiin kaigiroku dai 13 gô (registres parlementaires, chambre des représentants).

4. Selon ces accusations, le Premier ministre, tout en promettant à ses concitoyens durant toute la durée des négociations la non-ouverture du marché du riz, aurait en fait négocié secrètement une ouverture partielle de ce marché avec les États-Unis et les services concernés du GATT... 
ne peut ressentir que de l'indignation. Jusqu'ici l'émotion a pour origine l'expression des sentiments personnels du locuteur, aucun symbole de condensation n'a été employé. Le discours se poursuit par des arguments rationnels: les négociations agricoles de l'Uruguay Round ont tourné à l'avantage des grands pays exportateurs; la préservation de l'autosuffisance alimentaire est une priorité...

À la suite, le député du Parti libéral démocrate cite en exemple la France, qui réussira à l'issue de ces négociations à préserver son secteur audiovisuel de la libéralisation. Y. Mori explique que protéger sa culture, c'est protéger son originalité. Il se livre ensuite à un inventaire des liens existant entre le riz et la culture japonaise, aligne les symboles de condensation : évocation des cérémonies impériales de repiquage du riz, des fêtes religieuses dans les sanctuaires inari, lieux de culte dédiés au renard, divinité protectrice des moissons... Y. Mori conclut sur le riz en tant que patrimoine culturel national au même titre que l'audiovisuel pour la France. Sur les registres parlementaires, des applaudissements sont signalés uniquement à deux endroits de la longue intervention du député Mori; la fin du passage qui vient d'être évoqué est l'un de ces endroits.

Ainsi, Y. Mori a-t-il débuté son intervention sur des propos qui ont su stimuler l'imagination, ainsi que toucher son auditoire. Il n'a pourtant pas continué sur cette lancée. En effet le député conservateur, dans la deuxième partie de son discours, n'utilise que des arguments rationnels et n'évoque que des questions techniques.

Finalement, l'intervention sort de la technicité par la dénonciation virulente de la faiblesse d'un Premier ministre à qui «les intérêts» et «l'existence» d'un «peuple de plus de 120 millions d'âmes» ont été confiés. Y. Mori s'inquiète aussi de l'augmentation de la population mondiale, et de ses conséquences sur le régime alimentaire de son pays, passage ponctué par la deuxième et dernière salve d'applaudissements. Mais, au lieu d'achever son discours sur ce passage qui a su réveiller l'assistance, le député conservateur conclut par une énumération des différents échecs du gouvernement en place.

Un pathos trop concentré en début de discours, une surabondance de passages techniques paraissent d'un point de vue rhétorique être les principales faiblesses dans une perspective aristotélicienne:

En face de certains auditeurs, lors même que nous posséderions la science la plus précise, il ne serait pas facile de communiquer la persuasion par nos paroles à l'aide de cette science. Un discours scientifique tient de la doctrine, ce qui est (ici) d'une application impossible, attendu que pour produire des preuves et des 
raisons, il faut s'en tenir aux lieux communs, comme nous l'avons déjà dit dans les Topiques, à propos de la manière de parler à la multitude 5 .

\section{Le rejet du pathos ou la tyrannie de la raison}

Selon Aristote un discours politique composé uniquement d'arguments objectifs ne pourra avoir sur un large auditoire qu'un impact relativement faible. Si de plus l'orateur est inconnu, l'attention portée au discours sera quasiment nulle, confirme sur ce point Gustave Le Bon: «L'orateur inconnu arrivant avec un discours rempli de bonnes raisons, mais seulement de raisons, n'a aucune chance d'être seulement écouté ${ }^{6}{ }^{\prime}$.

Le président du parti socialiste japonais, Tomiichi Murayama, n'était pas un inconnu, mais son discours ne comportait que de bonnes raisons: la préservation de l'autosuffisance alimentaire et de l'environnement; le soutien nécessaire au gouvernement de coalition dont le parti socialiste constituait à cette époque la force politique la plus importante; la nécessaire réussite des négociations de 1'Uruguay Round ${ }^{7}$. Le discours fut de surcroit prononcé au petit matin, devant une assistance à moitié assoupie, à la capacité d'attention probablement très basse. Aussi est-il fort probable que l'impact des mots fut extrêmement réduit.

T. Murayama n'utilisa donc pas le moindre argument à caractère émotionnel, que ceux-ci aient été fondés sur l'expression des sentiments personnels du locuteur (regrets, honte...) ou sur des attaques ou condamnations de l'action du Gouvernement (accusations de traitrise, d'incompétence, etc.) ou encore sur des références mythico-symboliques de condensation (du type «le riz et le Japon, c'est 2000 ans d'histoire! »). T. Murayama aurait cependant reconnu pleinement ses responsabilités à l'occasion d'une conférence de presse donnée dans l'enceinte du Parlement, et y aurait également exprimé ses regrets de ne pas être parvenu à faire respecter les engagements électoraux de son parti (la non-ouverture du marché du riz). Cependant, seul le discours analysé ici fit l'objet d'une diffusion dans les médias, ainsi que dans l'organe officiel du parti.

Le parti socialiste était le parti de la coalition au pouvoir dont la posi-

5. Aristote, 1991, La rhétorique, Librairie Générale Française, p. 80.

6. G. Le Bon, 1947 ( $1^{\text {re }}$ édition 1895), Psychologie des foules, Presses Universitaires de France, p. 128.

7. 17 décembre 1993, Shakai shimpô («Nouvelles Sociales», journal du parti socialiste). 
tion était la plus délicate. Face à la possibilité de l'acceptation par le Gouvernement de la libéralisation partielle (bubun kaihô) du marché du riz, il hésita jusqu'à la date butoir des négociations de l'Uruguay Round (15 décembre 1993) entre deux attitudes possibles ${ }^{8}$. La première consistait à respecter les engagements électoraux de refus de l'ouverture du marché du riz et à quitter le Gouvernement, mais cette attitude avait l'inconvénient de conduire à la fin de l'expérience du gouvernement de coalition Hosokawa ${ }^{9}$. La seconde attitude consistait à maintenir le parti dans la coalition, et permettre ainsi la survie du Gouvernement, mais passait par le reniement des engagements électoraux et donc par une perte de confiance de l'opinion publique. Aussi, la brièveté et l'absence d'émotion de l'intervention de Murayama peut-elle s'expliquer à première vue par cette situation évidente d'embarras politique. Un embarras similaire, moins marqué toutefois, n'a pourtant pas empêché le Premier ministre, Morihiro Hosokawa, de prononcer un discours de conférence de presse très émouvant, comme il sera exposé un peu plus loin.

À l'occasion d'un entretien privé, en aout 2000, nous avons posé la question suivante à l'ancien président du parti socialiste:

Pourquoi avoir fait un discours aussi dépourvu de chaleur (tsumetai hatsugen)? Pourquoi, comme le Premier ministre Hosokawa, n'avoir pas exprimé votre douleur, ou encore, pourquoi ne pas avoir, à l'image toujours du Premier ministre, présenté des excuses à vos concitoyens?

T. Murayama nous a répondu en ces termes:

Cela s'explique par une différence de position. Dans mon cas, en tant que responsable de parti politique, je devais devenir le représentant des agriculteurs et me faire le porte-parole de leurs revendications, alors que dans celui d'Hosokawa, il s'agissait de faire face aux citoyens et de leur expliquer la politique du pays. Je pense que mon rôle était de demander au Gouvernement une politique agricole solide et responsable. En d'autres termes, je ne pouvais pas me permettre d'utiliser de belles expressions creuses (amai kotoba).

8. La proposition de compromis Denis (du nom de son auteur), soumise au Gouvernement japonais par les bureaux du GATT dans les dernières semaines précédant la fin des négociations, prévoyait pour le riz japonais le report à six années des négociations sur la mise en place d'un système de tarification douanière, mais imposait en contrepartie l'importation annuelle obligatoire d'une certaine quantité de riz, c'est-à-dire la libéralisation partielle du marché.

9. À l'occasion des élections législatives de juillet 1993 le paysage politique japonais fut profondément modifié. Les conservateurs du parti libéral démocrate (PLD), à la tête de l'État sans discontinuité depuis 1955 , perdaient le pouvoir au profit d'une coalition hétéroclite de huit partis politiques allant du parti socialiste à des partis de centre droit. 
La position défendue par T. Murayama nous semble caractéristique d'une conception très répandue dans les pays industrialisés, et tout particulièrement au Japon, de ce que doit être le comportement d'un homme politique. M. Edelman formule cette conception de la manière suivante:

Dans une culture dans laquelle science et logique sont honorées en tant que voies de la vérité, on doit s'attendre à ce que l'État, peut-être notre plus efficace et universel symbole, insiste sur l'appel à la raison à la fois dans ses procédures parlementaires et dans les justifications judiciaires officielles de ses décisions ${ }^{10}$.

En d'autres termes, les hommes politiques doivent si possible se garder de tout sentimentalisme. Dans le cas de T. Murayama cet attachement au pragmatisme le plus pur est tel que lors de notre entretien, la pertinence, voire le sens des questions relatives aux dimensions symboliques ou émotionnelles de la crise du riz lui furent à peu près incompréhensibles.

\section{Le pathos involontaire}

La députée communiste Sumi Fujita ne fit usage dans son discours d'aucune référence mythico-symbolique de condensation ${ }^{11}$. L'émotion fut pourtant très présente. Le pathos généré par la députée Fujita trouva son origine dans des expressions où perçaient colère et indignation. Elle affirma ainsi par exemple qu'elle ne pourrait jamais pardonner l'acte de trahison (haishinkôi) perpétré par le Premier ministre à l'égard de la volonté populaire. Elle condamna également l'attitude servile (hikutsu) du Premier ministre face aux pressions américaines. La source de l'émotion est le locuteur lui-même: la colère, l'indignation, la honte de la députée sont exprimées avec suffisamment de conviction pour être communicatives, déclenchant systématiquement les applaudissements (la députée communiste a été applaudie treize fois en cours d'intervention).

Et pourtant, malgré toute l'émotion perceptible dans son discours, S. Fujita nous expliqua que le problème de l'ouverture du marché du riz ne constituait pas un problème émotionnel ${ }^{12}$. Elle justifia son point de vue en nous expliquant qu'il s'agissait d'un problème qui touche à la culture,

10. Op. cit., p. 135.

11. 13 décembre 1993, Kampô (Journal officiel), gôgai, dai 128 kai kokkai, shûgiin kaigiroku dai 13 gô (registres parlementaires, Chambre des représentants).

12. Entretien accordé le 10 avril 1998. 
à l'avenir d'un peuple, mais qui peut tout à fait être abordé sans émotion. Son cas est d'autant plus symptomatique que dix minutes plus tôt, expliquant qu'il était douloureux de constater que la population des campagnes vieillissait, que les conditions d'existence de la plupart des agriculteurs étaient devenues difficiles, elle s'était mise à verser des larmes. Et c'est tout en pleurant qu'elle avait poursuivi :

Ce genre de situations difficiles est fréquent. Ce n'est pas une bonne chose que les paysans qui sont à la source de la vie (inochi no minamoto de aru) soient dans de telles situations...

Comment est-il possible de rendre compte de cet écart entre paroles, actes et surtout sentiments?

À cette question nous répondrons tout d'abord que de tous les partis japonais, le parti communiste apparait souvent comme le plus soucieux de rationalité et d'objectivité. Les discours, comme les documents issus de ce parti sont en général bien illustrés, remplis de statistiques et graphiques. La stratégie de communication est souvent fondée sur des preuves issues d'études scientifiques, c'est-à-dire chiffrée ${ }^{13}$. Cela très certainement dans le but de lutter contre une image de parti utopiste qui lui est souvent attribuée par l'opinion publique japonaise. Ce souci d'objectivité, voire de scientificité a notamment pour conséquence la proscription du pathos dans les communications officielles.

Examinons à la suite la réponse que fit $\mathrm{S}$. Fujita à la question suivante. «Pour quelles raisons, à l'occasion du discours que vous avez prononcé devant le Parlement, n'avez-vous pas utilisé d'images relatives à la tradition ou à l'identité japonaise? Vos arguments ont été essentiellement de type rationnel...». À cette question elle nous a répondu tout d'abord: «C'est parce que mon intervention était limitée dans le temps et parce qu'il s'agissait d'une tribune politique (seiji butai)», et a ajouté ensuite: «Ce genre de choses (les liens avec la tradition ou l'identité japonaise) est compris et connu. Il n'est pas nécessaire de le rappeler aux électeurs ». Cependant, en quoi une «tribune politique» interdirait-elle l'expression d'arguments liés à la tradition ou à l'identité? Ces liens sont-ils par

13. Voir notamment l'article du secrétaire général du Parti à cette époque, Kazuo Shii, «Nihon no kome o mamoru tame no nihon kyôsantô no itsutsu no kinkyû teian (Les cinq propositions d'urgence du Parti communiste japonais pour protéger le riz japonais)», Zen'ei, 665, 10 octobre 1995, p. 527-534, qui cite par exemple à l'appui de ses propos des évaluations chiffrées du rôle joué par les rizières dans la purification de l'eau et la préservation des inondations. 
ailleurs vraiment de telles évidences qu'il ne soit pas nécessaire de les rappeler? La représentante du parti communiste, soucieuse de ne produire que des arguments objectifs, et dans une attitude qui nous a paru symptomatique de la classe politique japonaise dans son ensemble, escamota de manière plus ou moins consciente de son discours devant le Parlement toutes les références possibles à l'héritage culturel japonais, c'est-à-dire tout «symbole de condensation» potentiel. Cependant, sa tentative théorique, héroïque, de gommer les aspects symboliques, c'est-à-dire émotionnels des enjeux, de n'en garder que les aspects rationnels fut au bout du compte un échec pratique: S. Fujita ne parvint pas à maitriser son émotion qui se traduisit par un ton excessivement véhément à l'occasion de sa question au Gouvernement, et par des larmes au moment de notre entretien...

\section{De l'importance rhétorique de l'usage équilibré du pathos et de la raison}

À l'occasion d'une conférence de presse, prononcée le 14 décembre 1993, c'est-à-dire au paroxysme de la crise du riz, le Premier ministre japonais de l'époque, Morihiro Hosokawa, fit preuve d'une grande maitrise rhétorique ${ }^{14}$.

[Relève de la rhétorique], tout discours qui joint l'argumentation au style, tout discours où les trois fonctions de plaire, d'instruire et d'émouvoir sont présentes ensemble et chacune par les autres; tout discours qui persuade par le plaisir et l'émotion en les soutenant par l'argumentation ${ }^{15}$.

Le texte de M. Hosokawa est remarquable dans son utilisation conjuguée de l'émotion et de la raison, dans le but à la fois de plaire, d'émouvoir et d'instruire. La composition de son texte répond en outre parfaitement aux règles de composition définies par la rhétorique classique, à savoir, quatre parties: exorde, narration, confirmation, péroraison.

Notons que la composition quadripartite d'un texte est également recommandée dans la poésie classique japonaise. Cette composition d'origine chinoise, appelée kishôtenketsu, a fortement influencé la littérature japonaise. Ses quatre parties, $k i$, l'entrée en matière, shô, le dévelop-

14. 14 décembre 1993, «Hosokawa shushô no hatsugen-bun», Mainichi shimbun («Journal Mainichi»), édition du soir, p. 3.

15. O. Reboul, 1998, La rhétorique, PUF, p. 32-33. 
pement, ten, le changement de perspective, ketsu, le résumé conclusif, présentent d'étonnantes similarités avec la structure de la rhétorique classique. Enseigné dès l'école primaire, le kishôtenketsu continue aujourd'hui encore de marquer non seulement la poésie mais également la prose japonaise.

Rappelons, par ailleurs, que le Japon moderne a emprunté à l'Occident l'essentiel de ses institutions et pratiques politiques. Dans les clubs universitaires de rhétorique, par où sont passés bon nombre de politiciens japonais, l'art occidental du discours politique est enseigné et pratiqué depuis le début du $20^{\mathrm{e}}$ siècle $^{16}$.

Aussi, le discours du Premier ministre japonais nous apparait-il comme le fruit de cette double influence: influence ancienne, littéraire de la Chine, moderne, politique de l'Occident. Ces remarques étant faites, examinons à présent dans le détail la conférence de presse du Premier ministre.

\section{L'exorde (ki)}

Les rhéteurs attribuaient à l'exorde la fonction de capter l'attention du public, «s'il doit annoncer le sujet, sa fonction principale est de plaire ${ }^{17}$. Le Premier ministre japonais n'a sacrifié à aucune entrée en matière. Il aborde immédiatement le vif du sujet:

J'ai en ce jour officiellement décidé d'accepter la proposition de compromis concernant l'accord final du volet agricole des négociations du GATT, Uruguay Round [c'est-à-dire la libéralisation partielle du marché du riz].

Cependant l'absence d'exorde est familière du style délibératif, c'est-àdire du discours politique. Si le public n'est pas séduit, son attention est du moins captée, car il est question immédiatement de l'essentiel: la proposition de compromis a finalement été acceptée, le marché du riz japonais fera l'objet d'une ouverture partielle. Le Premier ministre a ainsi délibérément choisi de commencer son discours par la conclusion.

\section{La narration (shô)}

La narration, qui suit l'exorde, doit être consacrée à l'exposé des faits.

16. Le club de l'université Waseda (waseda daigaku yûbenkai) est le plus célèbre des clubs de rhétorique.

17. O. Reboul, op. cit., p. 24. 
«Sa fonction première est d'instruire ${ }^{18}$. Aussi, M. Hosokawa fait-il tout d'abord un rapide historique avant d'expliquer les enjeux et les objectifs des négociations de l'Uruguay Round: "Il n'est pas exagéré de dire que de l'aboutissement de ces négociations dépend le destin du système commercial libéral, voire même de l'économie mondiale». Dans un souci didactique évident, il explique que ces négociations ont pour objectif d'établir une première réglementation du commerce international des produits agricoles. C'est vers la fin de la narration que se situe le premier passage émotionnel de la conférence du Premier ministre: «L'histoire du riz et l'histoire du Japon sont une seule et même chose»; «les épis de riz parvenus à maturité, comme les fleurs de cerisiers constituent depuis les temps les plus anciens les symboles de l'archipel japonais». Ces évocations mythico-historiques, le symbole de condensation constitué par «l'image des fleurs de cerisier» viennent à propos pour émouvoir l'auditoire après l'avoir instruit des faits.

Le Premier ministre termine sa narration par un exposé clair et détaillé des conditions proposées par l'accord de compromis qu'il vient d'accepter. Le public a été suffisamment instruit des faits et déjà quelque peu ému, il faut à présent justifier les positions adoptées; ce sera l'objet de la partie du discours que les anciens désignaient par le terme de «confirmation».

\section{La confirmation (ten)}

Comme la narration, le rôle de la confirmation est également d'instruire. La confirmation du Premier ministre occupe la place centrale de son discours. M. Hosokawa reconnait tout d'abord que l'accord de compromis accepté ne reflète qu'imparfaitement la position d'intransigeance défendue par le Japon (puisqu'elle introduit une libéralisation partielle du marché du riz). Mais, justifie-t-il, les négociations de l'Uruguay Round ont impliqué un grand nombre de pays avec des demandes et des attentes souvent difficilement conciliables. Aussi les bureaux du GATT ont-ils élaboré in extremis une proposition d'accord qui puisse à peu près convenir aux exigences de chacun. Le style de la conférence de presse prend un tour de plus en plus didactique.

La narration contient cependant un élément rhétorique essentiel dans l'économie générale du discours: une digression. Celle-ci est un «récit, une méditation, une prosopopée, qui renforce la preuve par l'émotion » ${ }^{19}$.

18. Ibid., p. 25.

19. Ibid. 
Le Premier ministre affirme ainsi que, comme ses concitoyens, son attachement pour le riz et les villages agricoles est profond, et que, par conséquent, la décision d'ouverture partielle du marché du riz, qu'il a été obligé de prendre, constitua pour lui un «véritable déchirement (masa ni danchô no omoi)», « l'expérience la plus pénible qu'il lui ait été donné de faire». Le récit de cette douleur personnelle n'apporte rien à l'argumentation, elle permet cependant d'émouvoir.

Après avoir présenté ses plus sincères excuses, M. Hosokawa poursuit sa confirmation à l'aide de divers arguments; par exemple, le fait que le Japon était à l'origine des négociations de l'Uruguay Round, et que, par conséquent, il aurait mauvaise grâce à les faire échouer. Enfin, M. Hosokawa présente une fois encore, «du fond du cœur», ses excuses.

\section{La péroraison (ketsu)}

M. Hosokawa entame à présent la dernière partie de son discours: la péroraison. Celle-ci «résume le discours et le termine par un appel, en général pathétique; après le plaire et l'instruire, c'est le moment de l'émouvoir ${ }^{20}$.

M. Hosokawa débute sa péroraison par l'exposé d'une conviction intime: la décision qu'il vient de prendre - l'ouverture partielle du marché $\mathrm{du}$ riz japonais - est la meilleure, non seulement pour l'agriculture japonaise, mais également pour le monde et, affirme-t-il, les développements futurs lui donneront certainement raison.

Il propose ensuite de saisir cette occasion pour opérer une rénovation de l'agriculture (nôgyô ishin). Le terme utilisé par le Premier ministre pour exprimer l'idée de «rénovation» est le même que celui qui est généralement traduit par «restauration» dans l'expression Meiji Ishin, ou Restauration Meiji. Cette dernière expression désigne le rétablissement en 1868 du pouvoir impérial, la fin du système du shogunat, et surtout l'ouverture au monde du Japon après plus de deux siècles et demi d'isolement volontaire. La coïncidence n'est certainement pas fortuite. L'ouverture du marché du riz a en effet souvent été comparée pendant cette période à l'ouverture du Japon aux influences occidentales dans la deuxième moitié du $19^{\mathrm{e}}$ siècle. Ouverture qui conduisit à la fin de la société dite «féodale» et à la modernisation intensive du pays. En 1993, «rénovation agricole» signifiait ouverture du marché à la concurrence étrangère, modernisation

20. Ibid. 
de l'agriculture japonaise, et vraisemblablement dans l'esprit de bon nombre de Japonais, l'entrée dans l'ère de la mondialisation du pays. Ainsi, grâce à l'emploi du terme ishin, le Premier ministre est-il parvenu à dramatiser ses propos de manière discrète, presque imperceptible, mais certainement non moins efficace, comme l'indique G. Le Bon:

L'orateur, en communication intime avec la foule, sait évoquer les images qui la séduisent. S'il réussit, son but a été atteint; et un volume de harangues ne vaut pas les quelques phrases ayant réussi à séduire les âmes qu'il fallait convaincre ${ }^{21}$.

M. Hosokawa a employé dans sa péroraison à la fois de puissants symboles de condensation, "restauration (ishin)», et des expressions à résonance très moderne. En effet, le Premier ministre s'engage à faire tout ce qui est dans ses possibilités pour proposer une "vision de régénération» de l'agriculture nationale (nôgyô saisei no bijon). Le terme bijon que l'on trouve dans l'expression japonaise est le résultat de la japonisation du terme d'origine anglaise "vision». Le Premier ministre introduit par cet artifice une touche de modernité qui sert l'exposé de ses perspectives d'avenir.

Ensuite, M. Hosokawa énumère les principaux problèmes auxquels était alors confrontée l'agriculture japonaise. Mais, contrairement au président du parti socialiste, qui dans son communiqué officiel s'était arrêté à cette énumération, le Premier ministre achève son discours par une métaphore de l'accouchement, dans laquelle sont associées douleur et renaissance: «Cette peine (celle de l'ouverture du marché du riz) est celle d'un pays qui s'ouvre véritablement au monde, elle est comparable à l'épreuve de l'enfantement (jintsû no shiren)». M. Hosokawa dans le prolongement du climat dramatique produit par cette métaphore de la peine appelle à un partage de la douleur entre tous les citoyens. Douleur, explique-t-il, qui ne doit pas être laissée aux seuls agriculteurs. Indiquons par ailleurs qu'au Japon la capacité «d'endurer (gaman suru)» les épreuves est une qualité morale fort estimée, de même que l'aptitude au repentir public.

21. G. Le Bon, p. 45. 


\section{$*$ \\ $* \quad *$}

En guise de conclusion, nous aimerions nous appuyer sur le résultat d'un sondage d'opinion effectué à l'issue des négociations du GATT et, par ce biais, apporter un étai supplémentaire à la thèse défendue dans le présent article: un langage administratif, voire scientifique n'aura que peu de portée sur des individus mis en émoi. Le langage politique, s'il cherche à être efficace, se doit de mêler l'usage du pathos à celui de la raison; ceci tout particulièrement à l'occasion d'une crise en étroit rapport avec l'identité collective. À la question, «quelles sont vos impressions concernant la façon d'agir du Gouvernement japonais qui a décidé d'ouvrir le marché du riz?», 4\% des personnes interrogées jugèrent positivement l'action du Gouvernement, $73 \%$ répondirent que la chose était inévitable. Seules $11 \%$ ont indiqué que la façon d'agir du Gouvernement était intolérable, et $8 \%$ qu'elles avaient l'impression d'avoir été trompées ${ }^{22}$. Une bonne majorité des Japonais n'a donc pas tenu rigueur au Gouvernement de sa décision d'ouverture partielle du marché du riz. En d'autres termes, la politique menée par le Premier ministre semble avoir été du point de vue de son impact sur l'opinion publique un relatif succès. M. Hosokawa aurait déclaré en 1997 «qu'alors que le public considérait son Gouvernement comme un gouvernement de réformes politiques, il aimait personnellement à le considérer comme un gouvernement de libéralisation $d u$ riz»... ${ }^{23}$.

La conférence de presse analysée dans cette article n'a pas été bien sûr le seul facteur de cette réussite du Gouvernement Hosokawa, mais nous croyons qu'elle y a fortement contribué. En effet, à la différence de la plupart des discours politiques de l'époque, l'allocution du Premier ministre semble avoir su pleinement répondre aux attentes émotionnelles du peuple japonais : expression de la douleur, excuses publiques, nombreuses évocations mythico-symboliques de condensation. Grâce à sa faculté d'empathie avec les sentiments du moment - notamment inquiétude vis-à-vis

22. Sondage réalisé en décembre 1993 par Asahi Shimbun-sha, dont les résultats complets sont donnés dans yoron chôsa nenkan: zenkoku yoron chôsa no genkyô, naikakusôridaijin kambôkôhôshitsu, Okurashô insatsu-kyoku, 1994, p. 444.

23. Selon une interview effectuée par G. L. Curtis, 1999, The logic of Japanese politics. Leaders, institutions and the limits of change, New York, Columbia University Press, p. 134. 
d'une certaine érosion de l'identité collective - le Premier ministre japonais est parvenu à faire accepter à ses concitoyens l'ouverture du marché du riz; c'est-à-dire certaines conséquences quelque peu angoissantes de la libéralisation à l'échelle planétaire des échanges commerciaux.

\section{Corpus}

On trouvera les références complètes des textes du corpus dans les notes.

\section{13 décembre 1993}

Question au gouvernement de la députée communiste Sumi Fujita et du député conservateur Yoshirô Mori.

\section{14 décembre 1993}

Conférence de presse du Premier ministre Morihiro Hosokawa.

3. 17 décembre 1993

Communication officielle du président du parti socialiste japonais, Tomiichi Murayama.

4. 10 avril 1998

Entretien avec Sumi Fujita (députée du parti communiste japonais).

5. 31 aout 2000

Entretien avec Tomiichi Murayama (président du parti socialiste japonais en décembre 1993). 